\title{
Strong cosmic censorship in charged de sitter spacetime with scalar field non-minimally coupled to curvature
}

\author{
Hong Guo ${ }^{1, \mathrm{a}}$, Hang Liu ${ }^{1, \mathrm{~b}}$, Xiao-Mei Kuang ${ }^{2,3, \mathrm{c}}$, Bin Wang ${ }^{2,3, \mathrm{~d}}$ \\ ${ }^{1}$ School of Physics and Astronomy, Shanghai Jiao Tong University, Shanghai 200240, China \\ ${ }^{2}$ College of Physical Science and Technology, Center for Gravitation and Cosmology, Yangzhou University, Yangzhou 225009, China \\ ${ }^{3}$ School of Aeronautics and Astronautics, Shanghai Jiao Tong University, Shanghai 200240, China
}

Received: 1 August 2019 / Accepted: 20 October 2019 / Published online: 5 November 2019

(C) The Author(s) 2019

\begin{abstract}
We examine the strong cosmic censorship in the Reissner-Nordstrom-de Sitter (RN-dS) black hole by investigating the evolution of a scalar field non-minimally coupled to the curvature. We find that for the stable RN-dS black hole, with the increase of the coupling parameter, the violation of the strong cosmic censorship occurs at a larger critical charge ratio. But such an increase of the critical charge is suppressed by the increase of the cosmological constant. Different from the minimal coupling situation, it is possible to accommodate $\beta \geq 1$ in the near extremal black hole when the scalar field is non-minimally coupled to curvature. $\beta$ here is defined as $\beta \equiv-\frac{\operatorname{Im} \omega}{\kappa_{-}}$where $\kappa_{-}$is the surface gravity of Cauchy horizon and $\omega$ is the frequency of quasinormal modes. The increase of the cosmological constant can allow $\beta \geq 1$ to be satisfied for even smaller value of the coupling parameter. The existence of $\beta \geq 1$ implies that the resulting curvature can continuously cross the Cauchy horizon.
\end{abstract}

\section{Introduction}

It is well-known that the existence of the Cauchy horizon $(\mathcal{C H})$, i.e., the inner horizon of black holes implies the loss of determinism of the physics laws beyond the $\mathcal{C H}$. To rescue the determinism, Penrose proposed his famous Strong Cosmic Censorship (SCC) long ago. The SCC is based on the fact that the $\mathcal{C H}$ of realistic black holes formed dynamically in the asymptotically flat spacetime is inextendible due to the occurrences of the so-called mass-inflation induced by the blue shift amplification effect of the unavoidable time-dependent remnant fields propagating along the $\mathcal{C H}$. The presence of

\footnotetext{
a e-mail: gh710105@gmail.com

be-mail: hangliu@sjtu.edu.cn

c e-mail: xmeikuang@yzu.edu.cn

de-mail: wang_b@sjtu.edu.cn
}

this scenario relies essentially on the inverse power law decay of the fields in the exterior region of asymptotically flat black hole spacetime, which cannot compete with the aforementioned blue shift amplification [1].

However, when one considers the de Sitter $(\Lambda>0)$ black holes, the situation we discussed above will change dramatically. Because in an asymptotically de Sitter spacetime, the perturbations outside the black holes decay instead exponentially in the form of $e^{-\alpha t}$, where $\alpha$ stands for the spectral gap, determined by the relation $\alpha=\inf \left\{-\operatorname{Im}\left(\omega_{i}\right)\right\}$ over all possible quasi-normal modes (QNMs) $\omega_{i}$ [2]. This kind of exponential decay behavior could be fast enough to make that the inner horizon singularity so weak that the spacetime metric would be extendible beyond the $\mathcal{C H}$ as a weak solution to the Einstein field equation [3], leading eventually to the violation of the SCC. The fate of the SCC depends on the delicate competition between the exponential decay outside the black hole and the blue shift amplification along the $\mathcal{C H}$ in the interior region of black hole. In particular, corresponding to a linearized scalar perturbation, it was found that the SCC may be violated if the condition $\frac{\alpha}{\kappa_{-}}>\frac{1}{2}$ is satisfied [2] for all QNMs, where the $\kappa_{-}$is the surface gravity of the $\mathcal{C H}$. Whereas the SCC will be respected if the condition $\beta \equiv-\frac{\operatorname{Im} \omega}{\kappa_{-}}>\frac{1}{2}$ can not be satisfied by all QNMs in the sense that the scalar field can not be extended across the $\mathrm{CH}$. This amounts to saying that if there exists one mode which satisfies $\beta \equiv-\frac{\operatorname{Im} \omega}{\kappa_{-}}<\frac{1}{2}$, then the SCC will hold.

In order to examine the stability of the ReissnerNordstrom-de Sitter (RN-dS) black holes, the QNMs have been investigated at various aspects, including the charged scalar field perturbation in four dimension [4], and the gravitational perturbation in higher dimensions [5,6]. In particular, due to the above relation between QNMs and SCC, the QNMs of RN-dS black holes have recently attracted resurgent attentions [1,2,7-17] since the pioneering work [2]. The linear neutral massless scalar perturbation is studied respec- 
tively in dimension $d=4$ [2], and in higher dimensions [16]. As a result, SCC is found to be violated when the black hole parameters are taken in the near extremal regime. Such a violation of SCC becomes more severe under the coupled electromagnetic and gravitational perturbations [7]. The discussions have also been generalized to the charged massless/massive scalar perturbation, and it has been shown that the SCC will be saved from being violated provided that the field is charged properly, but there is still a parameter regime in which the violation of SCC occurs [1,9-11]. The similar result has also been obtained for the Dirac field perturbation $[13,18]$. In addition, The non-linear evolution of massless neutral scalar perturbation is considered in RN-dS space and it turns out that the SCC might not be saved by such nonlinear effects [19]. On the other hand, the SCC in lukewarm RN-dS and Martnez-Troncoso-Zanelli black hole spacetime under the non-minimally coupled massive scalar perturbation was investigated and it was shown that the validity of the SCC depends on the characteristics of the scalar field [15]. Last but not least, although the Kerr-de Sitter black holes share many similarities with the $\mathrm{RN}-\mathrm{dS}$ ones, it is found that there is no violation of SCC for the linear perturbations [12]. Later on, no violation is further extended to higher dimensional Kerr-dS backgrounds for minimally coupled fields [14], as well as the non-minimally coupled massive scalar field [17].

It was argued that minimal coupling is of limited value in the context of effective field theories, while non-minimally coupled interactions were appreciated in the general study in gravity [20]. Specially, plenty of studies have been done in this direction, such as the spontaneous symmetry-breaking effect in gravity [21],the scalar field models of dark energy [22-25] and the low-energy limits of the superstring theory [26-28]. Besides, it was addressed in [29] that non-minimum coupling is necessary to avoid causal pathologies in field propagation in a general curved background. On the other hand, it is obvious that instead of perturbation minimally coupled to curvature, a non-vanishing coupling term would apparently influence the spectrum of QNMs such that the decay speed of the perturbation will be affected. Then the question, whether the local energy of the scalar perturbation is finite or not at $\mathcal{C H}$, is not obvious and deserves to be figured out.

Thus, it is interesting and important to study SCC under non-minimally coupled scalar field. The evolution of a scalar field coupled to curvature in topological black hole spacetimes was investigated in [30]. So far all investigations on the SCC are limited to fields that are minimally coupled to curvature in $\mathrm{RN}-\mathrm{dS}$ spacetime, it is natural to generalize the study to the non-minimal coupling scalar field. The work [17] studied the SCC and the quasinormal resonance of nonminimal coupling scalar field in the Lukewarm ReissnerNordström-de Sitter black holes, however they set the charge and the mass of the RN-dS black holes to be equal to each other. In our present work, we will not impose any relations between black hole charge $Q$ and mass $M$, and we will disclose the general validity of the strong cosmic censorship in four-dimensional Reissner-Nordström-de Sitter black hole against non-minimal coupling massless neutral scalar field perturbations. Considering that in [2] the SCC for massless neutral scalar perturbation with minimal coupling has been investigated, it is interesting to reveal the rich influences of the coupling parameter on the perturbation stability and the validity of the SCC.

The rest of the work is organized as follows. In Sect. 2, we give a brief and general introduction of the $\mathrm{RN}-\mathrm{dS}$ spacetime in $d \geq 4$ dimensions and derive the basic equations which control the motion of linear scalar perturbations. In Sect. 3, we analyze the stability of RN-dS black holes under nonminimally coupled linear scalar perturbations to disclose the impact of coupling constant on the stability of the RN-dS spacetime. In Sect. 4, we would like to investigate the validity of the SCC under scalar perturbations with different coupling constants, and we conclude with some discussions in the last section.

\section{Scalar perturbations with non-minimal coupling and the relation between QNMs and SCC}

In this section, we would like to give a general derivation of the equation of motion of the scalar field in $d \geq 4$ dimensional $\mathrm{RN}-\mathrm{dS}$ background. The metric of $\mathrm{RN}-\mathrm{dS}$ spacetime in d-dimension $(d \geq 4)$ is given by [31]

$d s^{2}=-f(r) d t^{2}+\frac{1}{f(r)} d r^{2}+r^{2} d \Omega_{d-2}^{2}$,

where

$f(r)=1-\frac{m}{r^{d-3}}+\frac{q^{2}}{r^{2(d-3)}}-\frac{2 \Lambda}{(d-2)(d-1)} r^{2}$,

and

$\Lambda=\frac{(d-2)(d-1)}{2 L^{2}}, \quad d \Omega_{d-2}^{2}=d \chi_{2}^{2}+\prod_{i=2}^{d-2} \sin ^{2} \chi_{i} d \chi_{i+1}^{2}$,

in which $L$ is the cosmological radius associated with cosmological constant $\Lambda$, while the parameter $q$ and $m$ are related to the electric charge $Q$ and the ADM mass $M$ of the black hole as

$M=\frac{d-2}{16 \pi} \omega_{d-2} m, \quad Q=\frac{\sqrt{2(d-2)(d-3)}}{8 \pi} \omega_{d-2} q$, 
$\omega_{d}=\frac{2 \pi^{\frac{d+1}{2}}}{\Gamma\left(\frac{d+1}{2}\right)}$,

with $\omega_{d}$ being the volume of the unit d-sphere. Furthermore, we have the electromagnetic field $F$ and gauge potential $A$

$F_{a b}=(d A)_{a b}, \quad A_{a}=-\sqrt{\frac{d-2}{2(d-3)}} \frac{q}{r^{d-3}}(d t)_{a}$

The action of the scalar field with a coupling constant $\lambda$ coupled with constant Ricci curvature $\mathcal{R}=2 d /(d-2) \Lambda$ is

$S_{\psi}=-\frac{1}{2} \int \mathbf{d}^{d} x \sqrt{-g}\left(\overline{D_{\nu} \psi} D^{\nu} \psi+\left(\mu^{2}+\lambda \mathcal{R}\right)|\psi|^{2}\right)$,

by which the equation of motion and energy-momentum tensor of the scalar field in the $d$-dimensional curved spacetime can be obtained as

$$
\begin{aligned}
& \left(D^{\nu} D_{\nu}-\left(\mu^{2}+\lambda \mathcal{R}\right)\right) \psi=0 \\
& T_{\mu \nu}=\overline{D_{(\mu} \psi} D_{\nu)} \psi-\frac{1}{2} g_{\mu \nu}\left(\overline{D_{\rho} \psi} \partial^{\rho} \psi+\mu^{2}|\psi|^{2}\right) \\
& \quad+\lambda\left(g_{\mu \nu} \square-\nabla_{\mu} \nabla_{\nu}+G_{\mu \nu}\right)|\psi|^{2}
\end{aligned}
$$

where $G_{\mu \nu}$ is the Einstein tensor, the operator $D_{\nu}=\nabla_{\nu}-$ ie $A_{v}$ is the extended covariant derivative, while $e$ and $\mu$ are the electric charge and the mass of the field, respectively. We expand $\psi$ in the following way

$\psi(r, t, \theta)=\sum_{l m} e^{-i \omega t} \frac{\phi(r)}{r^{\frac{d-2}{2}}} Y_{l m}(\chi)$.

Whence the equation of motion reads

$$
0=f(r) f^{\prime}(r) \phi^{\prime}(r)+f^{2}(r) \phi^{\prime \prime}(r)+\left(B_{1}+B_{2}\right) \phi(r),
$$

in which

$$
\begin{aligned}
B_{1}= & \frac{(d-2) e^{2} q^{2} r^{2(3-d)}}{2(d-3)}-\frac{\sqrt{2} e q r^{3-d}}{\left(\frac{d-3}{d-2}\right)^{\frac{1}{2}}} \omega+\omega^{2} \\
= & (\omega-\Phi(r))^{2} \\
B_{2}= & f(r)\left(-\frac{l(d+l-3)}{r^{2}}-\mu^{2}-\frac{2 d}{d-2} \Lambda \lambda\right. \\
& \left.-\frac{(d-2) f^{\prime}(r)}{2 r}\right)-f^{2}(r) \frac{(d-4)(d-2)}{4 r^{2}},
\end{aligned}
$$

and

$\Phi(r)=\frac{q e}{\sqrt{\frac{2(d-3)}{d-2}} r^{d-3}}$.
In the present paper, we focus only on the massless neutral scalar perturbations in the four-dimensional RN-dS spacetime, which corresponds to $e=\mu=0$ and $d=4$. As a result, the equation can be simplified into

$f(r) f^{\prime}(r) \phi^{\prime}(r)+f^{2}(r) \phi^{\prime \prime}(r)+\left(\omega^{2}-V_{e f f}\right) \phi(r)=0$,

where

$V_{e f f}=f(r)\left(\frac{l(l+1)}{r^{2}}+4 \Lambda \lambda+\frac{f^{\prime}(r)}{r}\right)$

As one can see, the only effect of non-minimal coupling constant $\lambda$ is that the effective potential $V_{\text {eff }}$ will be affected by the term $4 \Lambda \lambda$. Note that we are considering the case with a positive cosmological constant $\Lambda$, thus a positive $\lambda$ will increase the value of $V_{\text {eff }}$ while a negative $\lambda$ will decrease $V_{\text {eff }}$ such that $V_{\text {eff }}$ will have a wider and deeper negative region, leading to a potential instability of the black hole spacetime, as we shall discuss in Sect. 3.

By introducing the tortoise coordinate $d r_{*}=\frac{d r}{f(r)}$, we finally arrive at the master equation in the form of a Schrodinger equation

$\frac{d^{2} \phi(r)}{d r_{*}^{2}}+\left(\omega^{2}-V_{e f f}\right) \phi(r)=0$,

which gives rise to the spectrum of QNMs once the boundary conditions are imposed as follows

$\phi(r) \approx e^{-i \omega r_{*}},\left(r \rightarrow r_{+}\right) ; \quad \phi(r) \approx e^{i \omega r_{*}},\left(r \rightarrow r_{c}\right)$.

To relate the QNMs to SCC, we may as well go to the ingoing coordinate $v=t+r_{*}$ outside the black hole. Because our QNMs behaving as $e^{-i \omega v}$ across the event horizon are analytic functions in this coordinate, we can analytically continue our QNMs solutions to the inside of the black hole. In the inside of the black hole, we can go back to the $t$ coordinate, where our analytically continued solutions behave as $e^{-i \omega t}$. One should note that generically we will have both the ingoing and outgoing modes at the $\mathcal{C H}$, i.e.,

$\phi_{\text {out }} \sim e^{i \omega r_{*}}, \quad \phi_{\text {in }} \sim e^{-i \omega r_{*}}, \quad r \rightarrow r_{-}$.

Taking into account that the ingoing coordinate $v$ is singular while the outgoing coordinate $u=t-r_{*}$ is well defined at the $\mathcal{C H}$, we would like to move onto the $u$ coordinate, where the two modes behave as

$$
\begin{aligned}
\phi_{\text {out }} & \sim e^{-i \omega u}, \quad \phi_{\text {in }} \sim e^{-i \omega u-2 i \omega r_{*}} \\
& \sim e^{-i \omega u}\left(r-r_{-}\right)^{\frac{i \omega}{\kappa_{-}}}, r \rightarrow r_{-} .
\end{aligned}
$$


Obviously, the non-smoothness comes solely from the $\phi_{i n}$ mode. the $\mathcal{C H}$ is extendible if and the energy-momentum tensor (2.8) consisting of terms like $\psi^{2}, \partial_{\mu} \psi \partial_{\nu} \psi$ and $\nabla_{\mu} \nabla_{\nu} \psi^{2}$ of the scalar field should be integrable near the $\mathcal{C H}$, which amounts to saying that $\int\left(r-r_{-}\right)^{2(p-1)} d r$ should be finite with $p=\frac{i \omega}{\kappa_{-}}$. This requirement leads to $2[\operatorname{Re}(p)-1]>-1$. Thus the criterion for the violation of the SCC is given by

$\beta=-\frac{\operatorname{Im} \omega}{\kappa_{-}}>\frac{1}{2}$

for all QNMs. This means that if we can find some modes which meet the condition

$\beta<\frac{1}{2}$,

then the SCC will be respected.

We note that it is subtle to make the strong cosmic censorship conjecture precise and one should be careful when mentioning "violation of SCC ". This is because the existence of different formulations of SCC depends on the different smoothness of perturbation on $\mathcal{C H}$ in literatures. Given that whether perturbations or observers can across the $\mathcal{C H}$ is determined by the existence of solutions to the equations of motion of the matter fields. Accordingly, the SCC may be formulated since the maximal Cauchy development should be inextensible as a solution to the equations of motion. One may note that the fields equations are second order, which implies that the smoothness of fields should be $C^{2}$. This is the $C^{2}$ formulation of SCC which states that the spacetime can not be extended across the $\mathcal{C H}$ with $C^{2}$ metric. Actually, as argued in Ref. [7], the generic perturbations arising from the smooth initial data can be arbitrarily smooth at the $\mathrm{CH}$ when the black holes are close enough to extremality as well as large enough in a dS spacetime. This leads to the potential violation of $C^{r}$ formulation of SCC with any $r \geq 2$. Besides, the existence of the so called weak solutions motivates the Christodoulou's formulation of SCC, which states that generically the maximal Cauchy development is inextensible as a spacetime with locally square integrable Christoffel symbols (More detail also see [7]).

It is worthwhile to point out that the criterion for the violation of the SCC in this work is derived by Christodoulou's formulation. It was proven that in Ref. [32] that for any smooth initial data, the scalar field is $C^{1}$ at the $\mathcal{C H}$ if $\beta>1$, and if $\beta>1 / 2$ then the local energy at $\mathcal{C H}$ of scalar field is finite. This indicates that the Christodoulou's formulation of SCC is violated in $\mathrm{RN}-\mathrm{dS}$ spacetime, while this version of SCC is respected if $\beta<1 / 2$. For $\beta<1$, the generic scalar perturbations in $\mathrm{RN}-\mathrm{dS}$ spacetime fail to be $C^{1}$, therefore, the blow up of Kretschmann scalar implies that the $C^{2}$ formulation of SCC is supported as the curvature tensor includes the second order derivative of metric. On the contrary, if $\beta \geq 1$, the scalar field is $C^{1}$ at $\mathcal{C H}$ resulting in finite curvature at $\mathcal{C H}$ which implies a more severe violation of SCC. This will be shown in our numerical results soon.

\section{Stability analysis}

Only for the stable black hole, the discussion of the cosmic censorship is meaningful. Thus before we investigate the strong cosmic censorship, we would first like to conduct a stability analysis of the $\mathrm{RN}-\mathrm{dS}$ black hole under the neutral massless non-minimally coupled scalar fields. By inspecting the effective potential (2.15), we can see that the effective potential is affected by the coupling constant $\lambda$ in the form of $4 \Lambda \lambda$. Whence we can infer that the instability may occur if $\lambda$ is negative with big enough magnitude no matter which angular number $l$ we choose. On the contrary, note that the $\mathrm{RN}-\mathrm{dS}$ black hole is stable under the neutral massless minimally coupled $(\lambda=0)$ scalar field [4], and the appearance of the positive coupling constant will increase the effective potential such that the stability of the black hole is reinforced. In the following discussion, we set $M=1$ for simply.

In Fig. 1, we show the behavior of effective potential $V_{e f f}$ between the event horizon $r_{+}$and the cosmological horizon $r_{c}$ for $l=0$ and $l=1$. As expected from the formula (2.15), the larger angular number $l$, the more negative coupling constant is needed to have the occurrence of negative effective potential $V_{\text {eff }}$ in some region. Such an occurrence signals the possible instability of the black hole $[33,34]$. To see what really happens, one is required to calculate the corresponding QNMs, where the positive imaginary part of QNM frequency $\omega=\omega_{R}+i \omega_{I}$ corresponds to the growing mode, indicating the instability of the black hole. In this work, the QNMs are computed by the asymptotic iteration method(AIM) of which a brief introduction can be seen in the Appendix. There also briefly do the analysis on numerical accuracy of AIM relative to spectral method for sample of our model parameters, so that our following numerical results are reliable.

After checking an amount of the QNMs data within negative coupling parameter, we list the $n=0$ QNMs with the angular number $l=0,1,5,10$ for negative and non-negative $\lambda$, respectively. Tables 1 and 2 respect our conclusion. For the negative $\lambda$, one can see there exists a purely imaginary unstable mode at $l=0$. Moreover, the less negative the coupling constant $\lambda$ is, the smaller the imaginary of part of this unstable mode is. While for $\lambda \geq 0$, the unstable mode does not exist, indicating that the black hole is stable.

\section{Strong cosmic censorship}

For the stable black hole configuration, whether the cosmic censorship can be respected is an interesting question to be 

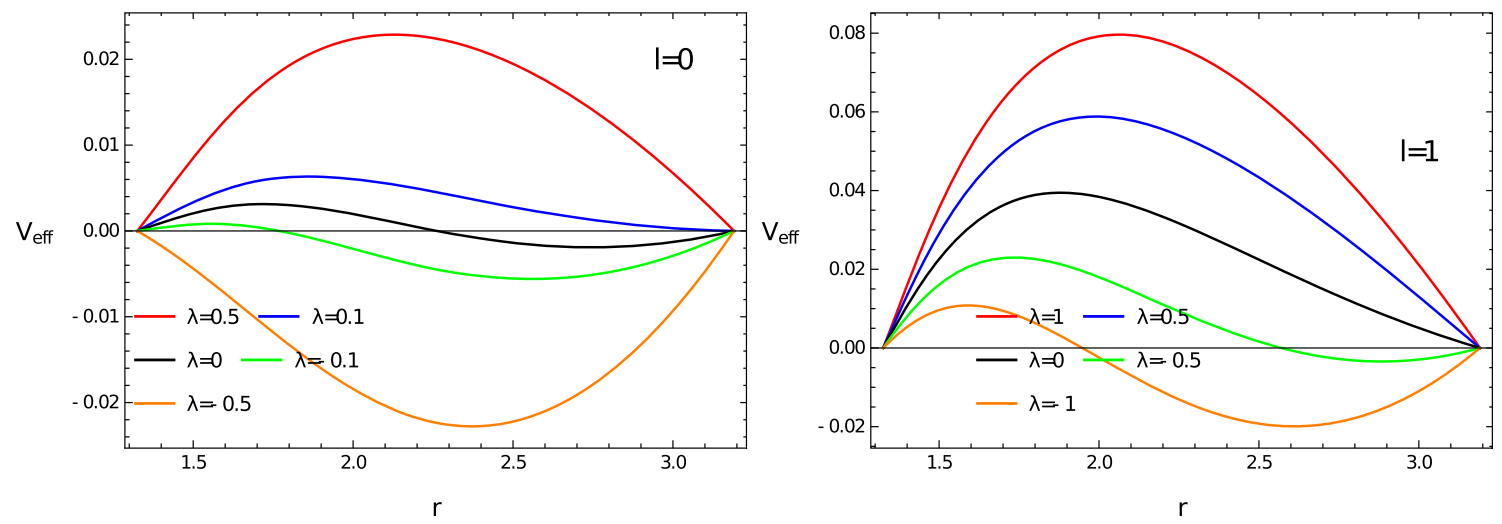

Fig. $1 M=1, \Lambda=0.14, Q / Q_{\max }=0.99$

Table 1 The QNMs for negative coupling constant when $M=1, \Lambda=0.14, Q / Q_{\max }=0.99, n=0$

\begin{tabular}{llll}
\hline Angular number & $\lambda=-0.5$ & $\lambda=-0.1$ & $\lambda=-0.001$ \\
\hline$l=0$ & $0+i 0.111107$ & $0+i 0.035139$ & $0+i 0.000470$ \\
$l=1$ & $0-i 0.007209$ & $0.181861-i 0.047444$ & $0.191424-i 0.046261$ \\
$l=5$ & $0.730575-i 0.045157$ & $0.740796-i 0.044912$ & $0.743318-i 0.044854$ \\
$l=10$ & $1.41688-i 0.044862$ & $1.42221-i 0.044798$ & $1.42352-i 0.044782$ \\
\hline
\end{tabular}

Table 2 The QNMs for positive coupling constant when $M=1, \Lambda=0.14, Q / Q_{\max }=$ $0.99, n=0$

\begin{tabular}{llll}
\hline Angular number & $\lambda=0$ & $\lambda=0.1$ & $\lambda=0.5$ \\
\hline$l=0$ & $0.041017-i 0.069190$ & $0.057096-i 0.049195$ & $0.143892-i 0.043464$ \\
$l=1$ & $0.19152-i 0.046245$ & $0.201098-i 0.045302$ & $0.237539-i 0.043293$ \\
$l=5$ & $0.743344-i 0.044854$ & $0.745888-i 0.044796$ & $0.756032-i 0.044575$ \\
$l=10$ & $1.42354-i 0.044782$ & $1.42487-i 0.044766$ & $1.43019-i 0.044704$ \\
\hline
\end{tabular}

examined. In the following we will investigate the strong cosmic censorship of the stable Reissner-Nordstrom-de Sitter (RN-dS) black hole by studying the evolution of a scalar field non-minimally coupled to the curvature.

\subsection{The fate of SCC}

Note that we always have an unstable mode for the negative coupling parameter, so regarding the potential violation of the SCC, below we shall focus only on the non-negative coupling parameter. We plot the most dominant modes for $l=0,1,2,10$ in Fig. 2, where each column corresponds to the result for the same cosmological constant with the coupling parameter increased from the top to the bottom, and the black vertical line is used to indicate the critical charge ratio $\frac{Q_{c}}{Q_{\max }}$ when $\beta$ arrives the critical value $\frac{1}{2}$.

As one can see, the violation of the SCC occurs no matter how we choose the value of the coupling parameter and cosmological constant. In addition, for a fixed cosmological constant, the aforementioned critical charge ratio increases with the coupling parameter. So the larger the coupling parameter is, the harder the violation of the SCC is. To scrutinize such a behavior, we plot the variation of the critical charge ratio as the increase of the coupling parameter in Fig. 3. First, it seems that such an increase will be saturated at a large coupling parameter. That amounts to saying that a larger coupling parameter will not affect the critical charge for the violation of the SCC any more. Second, the larger the cosmological constant is, the tinier such an increase is. In this sense, the cosmological constant seems to play a role in refraining the effect of the coupling parameter onto the critical charge.

\subsection{The blow up of the curvature}

It has been shown in [7] that $\beta<1$ for all the modes in the case of the minimal coupling, so the curvature blows up although the $\mathcal{C H}$ is extendible. Note that our scalar is coupled with the curvature, thus it is interesting to ask whether the curvature can cross the $\mathcal{C H}$ continuously when the coupling parameter is non-vanishing.

As such, we list the most dominant QNM data at each angular number in Tables 3, 4 and 5 for the charge ratio $\frac{Q}{Q_{\max }}=0.9990244$, where the most dominant mode is blackened. As we see, $\beta \geq 1$ can happen to all modes when the 

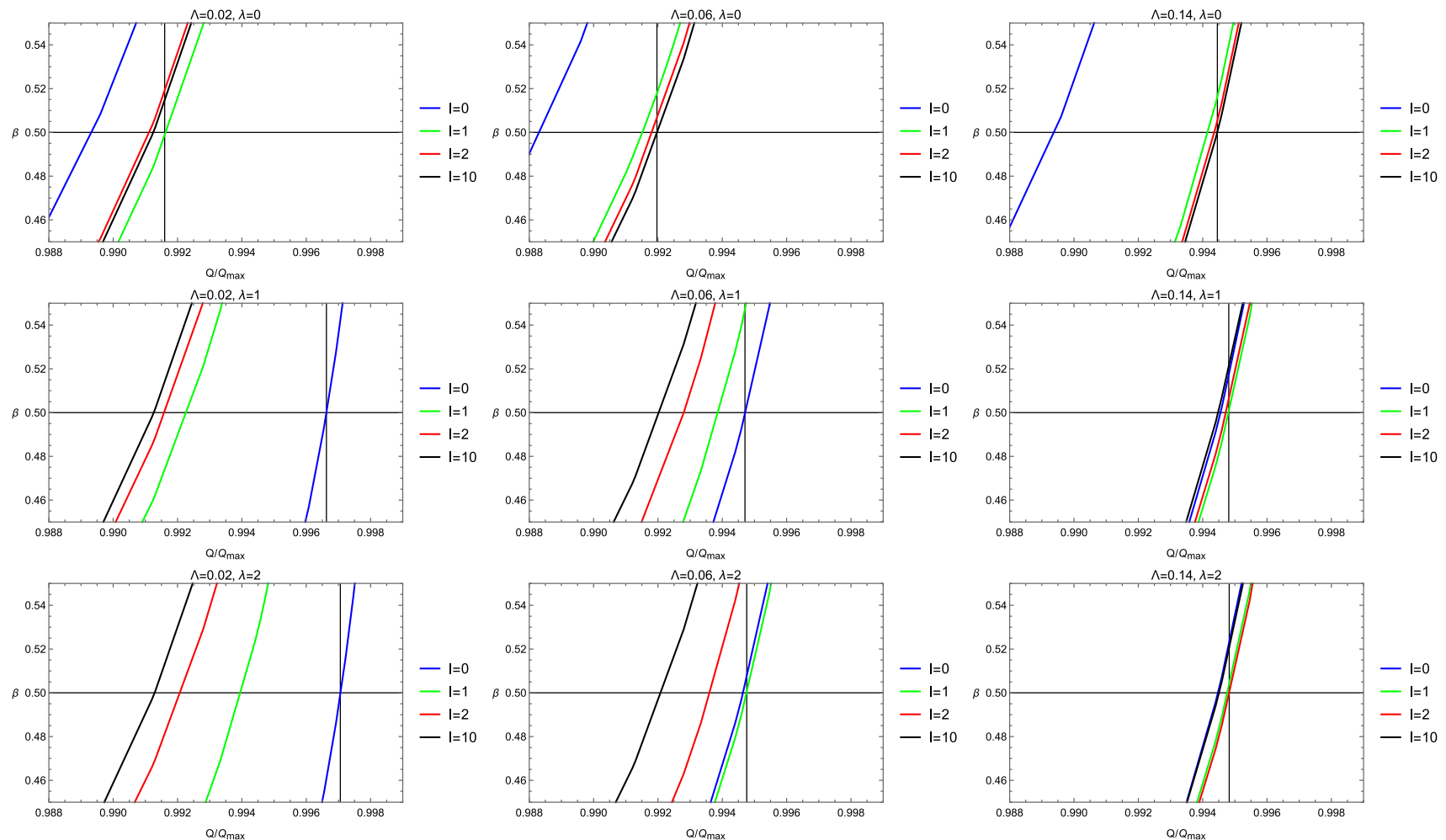

Fig. 2 The figures show how the different dominant modes cross through the horizontal black line $\beta=0.5$ in the different coupling parameters $\lambda=0,1,2$ when we fix the cosmological constant

$\Lambda=0.02,0.06,0.14$. The critical charge ratio $\frac{Q_{c}}{Q_{\max }}$ is determined by the intersection of the black vertical line with the horizontal line
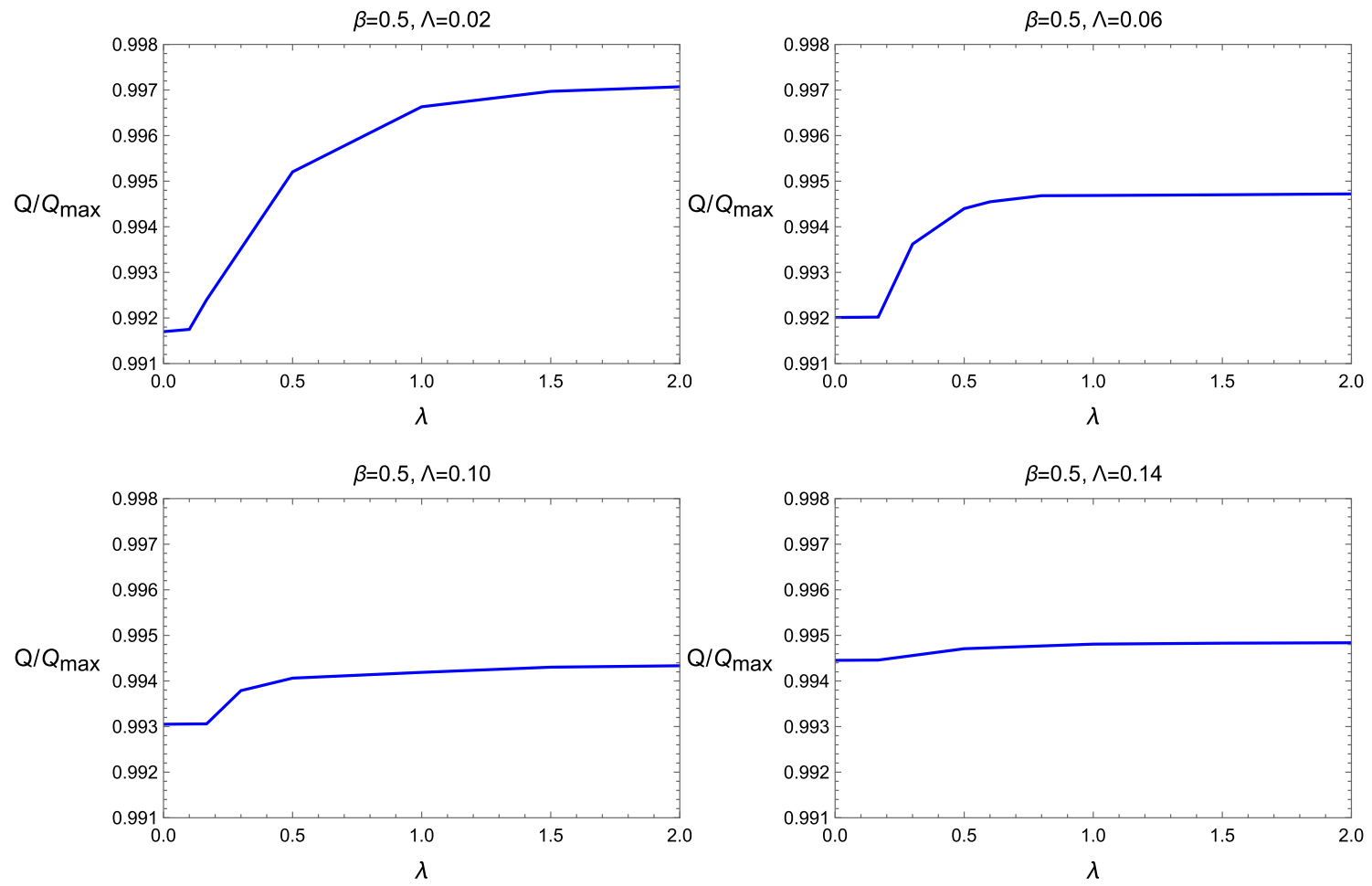

Fig. 3 The variation of the critical charge ratio $\frac{Q_{c}}{Q_{\max }}$ with the increase of the coupling parameter 
Table 3 The most dominant QNMs $\frac{\omega}{\kappa_{-}}$at each angular number in the case of $\Lambda=0.06, \frac{Q}{Q_{\max }}=0.9990244$ for $\lambda=0,1,2$

\begin{tabular}{lllr}
\hline$\frac{\omega}{\kappa_{-}}$ & $\lambda=0$ & $\lambda=1$ & $\lambda=2$ \\
\hline$l=00$ & $\mathbf{0 . 0}-i \mathbf{0 . 9 1 0 2 9 6}$ & $\mathbf{0 . 0}-i \mathbf{1 . 1 3 5 3 6 6}$ & $\mathbf{8 . 5 2 0 3 7 7 - i \mathbf { 1 . 3 4 1 3 3 0 }}$ \\
$l=01$ & $7.313771-i 1.777196$ & $8.904893-i 1.452243$ & $10.538373-i 1.319612$ \\
$l=02$ & $12.363690-i 1.731134$ & $13.289741-i 1.605510$ & $14.250843-i 1.490133$ \\
$l=03$ & $17.378690-i 1.718803$ & $18.036803-i 1.655218$ & $18.706578-i 1.592246$ \\
$l=04$ & $22.381133-i 1.713772$ & $22.892211-i 1.675494$ & $23.408555-i 1.637223$ \\
$l=05$ & $27.377763-i 1.711241$ & $27.795629-i 1.685680$ & $28.216331-i 1.660093$ \\
$l=06$ & $32.371227-i 1.709786$ & $32.724677-i 1.691516$ & $33.080409-i 1.673191$ \\
$l=07$ & $37.362790-i 1.708877$ & $37.669058-i 1.695166$ & $37.976397-i 1.681435$ \\
$l=08$ & $42.353112-i 1.708271$ & $42.623293-i 1.696914$ & $42.894239-i 1.686921$ \\
$l=09$ & $47.342596-i 1.707848$ & $47.584302-i 1.699309$ & $47.826571-i 1.690759$ \\
$l=10$ & $52.331439-i 1.707537$ & $52.549653-i 1.700606$ & $52.769209-i 1.693527$ \\
\hline
\end{tabular}

Table 4 The most dominant QNMs $\frac{\omega}{\kappa_{-}}$at each angular number in the case of $\Lambda=0.10, \frac{Q}{Q_{\max }}=0.9990244$ for $\lambda=0,1,2$

\begin{tabular}{llll}
\hline$\frac{\omega}{\kappa_{-}}$ & $\lambda=0$ & $\lambda=1$ & $\lambda=2$ \\
\hline$l=00$ & $\mathbf{0 . 0}-i \mathbf{0 . 8 9 4 8 0 4}$ & $\mathbf{0 . 0}-i \mathbf{1 . 2 9 4 1}$ & $9.490167-i 1.467466$ \\
$l=01$ & $6.883138-i 1.653291$ & $9.2443874-i 1.429376$ & $\mathbf{1 1 . 3 3 2 7 6 5 - i \mathbf { 1 . 4 0 2 5 9 3 }}$ \\
$l=02$ & $11.792684-i 1.606922$ & $13.215042-i 1.497977$ & $14.618450-i 1.434143$ \\
$l=03$ & $16.632350-i 1.595061$ & $17.644728-i 1.535904$ & $18.656848-i 1.487504$ \\
$l=04$ & $21.449082-i 1.590290$ & $22.235217-i 1.553812$ & $23.022197-i 1.520846$ \\
$l=05$ & $26.255466-i 1.587899$ & $26.898147-i 1.563277$ & $27.541511-i 1.540116$ \\
$l=06$ & $31.056298-i 1.586531$ & $31.599857-i 1.568825$ & $32.143897-i 1.551832$ \\
$l=07$ & $35.853795-i 1.585678$ & $36.324751-i 1.572329$ & $36.796042-i 1.559400$ \\
$l=08$ & $40.649209-i 1.585116$ & $41.064541-i 1.574717$ & $41.480342-i 1.564540$ \\
$l=09$ & $45.442989-i 1.584706$ & $45.814732-i 1.576372$ & $46.186605-i 1.568186$ \\
$l=10$ & $50.235561-i 1.584247$ & $50.572279-i 1.577484$ & $50.908526-i 1.570854$ \\
\hline
\end{tabular}

Table 5 The most dominant QNMs $\frac{\omega}{\kappa_{-}}$in the case of $\Lambda=0.14, \frac{Q}{Q_{\max }}=0.9990244$ for $\lambda=0,1,2$

\begin{tabular}{llll}
\hline$\frac{\omega}{\kappa_{-}}$ & $\lambda=0$ & $\lambda=1$ & $\lambda=2$ \\
\hline$l=00$ & $\mathbf{0 . 0}-i \mathbf{0 . 8 6 5 4 7 3}$ & $6.692527-i 1.401811$ & $9.562236-i 1.415554$ \\
$l=01$ & $6.295308-i 1.445910$ & $\mathbf{9 . 0 4 6 7 7 0}-i \mathbf{1 . 3 4 9 1 1 3}$ & $11.273336-i 1.358708$ \\
$l=02$ & $10.917835-i 1.415302$ & $12.637447-i 1.360230$ & $\mathbf{1 4 . 2 3 8 8 5 2}-i \mathbf{1 . 3 4 3 5 5 0}$ \\
$l=03$ & $15.444360-i 1.407717$ & $16.682366-i 1.374939$ & $17.882357-i 1.355230$ \\
$l=04$ & $19.940636-i 1.404689$ & $20.906034-i 1.383618$ & $21.855045-i 1.367681$ \\
$l=05$ & $24.423416-i 1.403178$ & $25.214220-i 1.388650$ & $25.996507-i 1.376473$ \\
$l=06$ & $28.898990-i 1.402315$ & $29.568563-i 1.391740$ & $30.233139-i 1.382379$ \\
$l=07$ & $33.370262-i 1.401774$ & $33.950764-i 1.393755$ & $34.528132-i 1.386401$ \\
$l=08$ & $37.838760-i 1.401415$ & $38.351116-i 1.395128$ & $38.861309-i 1.389234$ \\
$l=09$ & $42.305368-i 1.401164$ & $42.763880-i 1.396114$ & $43.220798-i 1.391338$ \\
$l=10$ & $46.770491-i 1.401242$ & $47.185146-i 1.396836$ & $47.599524-i 1.393582$ \\
\hline
\end{tabular}



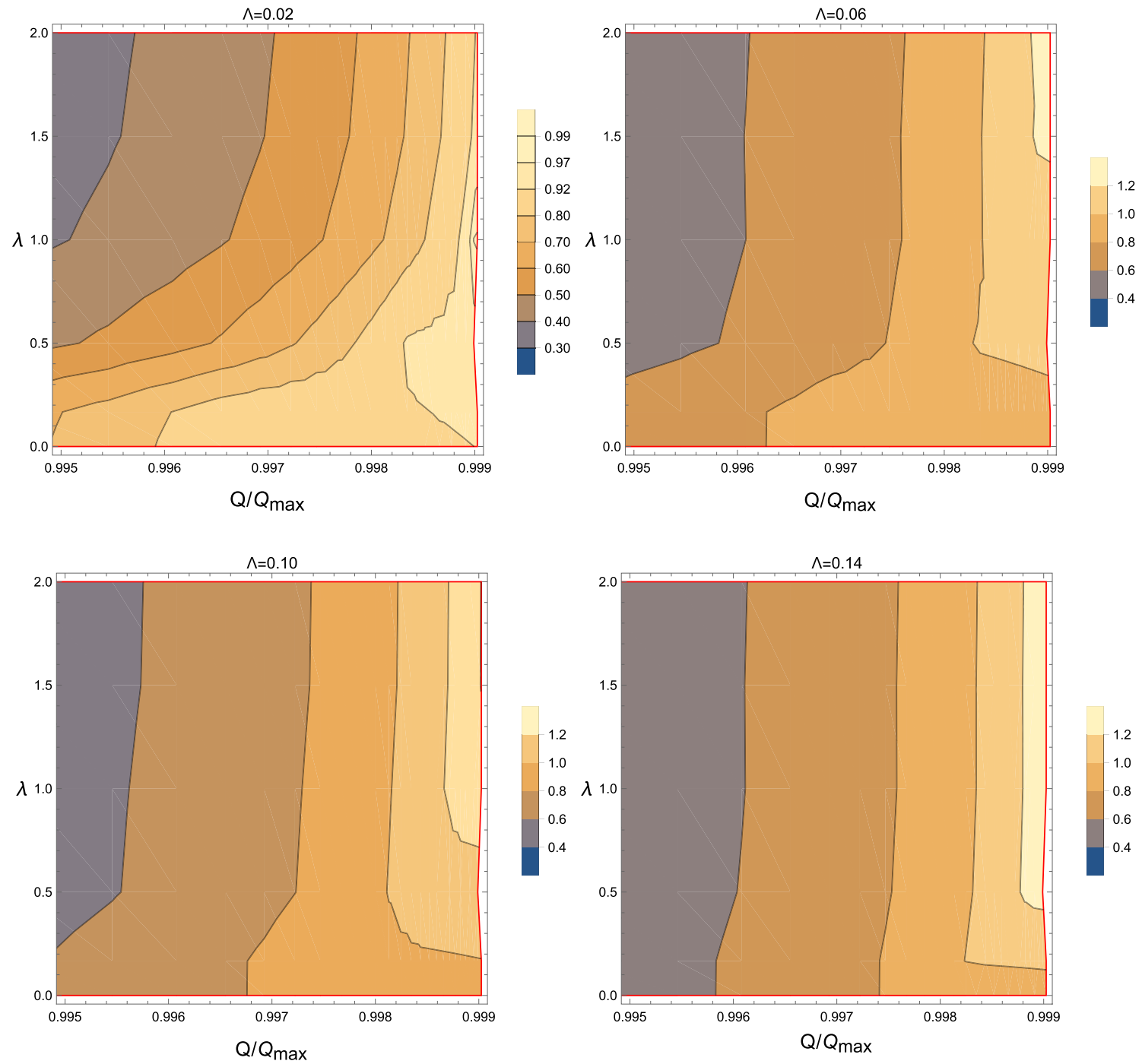

Fig. 4 The contour of $\beta$ for the most dominant mode in the space of the charge ratio $\frac{Q}{Q_{\max }}$ and coupling parameter $\lambda$

coupling parameter is non-vanishing. Furthermore, in Fig. 4, we plot the contour of $\beta$ for the most dominant mode in the space of the charge ratio $\frac{Q}{Q_{\max }}$ and coupling parameter $\lambda$. As one can see, when the cosmological constant is small, $\beta$ will not go beyond the limit $\beta=1$. When the cosmological constant is increased, the region of $\beta \geq 1$ emerges in the near extremal regime and the corresponding range of coupling parameter is enlarged toward the small values.

\section{Conclusions and discussions}

In this paper, we consider the perturbation of the nonminimally coupled scalar field in four dimensional $\mathrm{RN}-\mathrm{dS}$ spacetime. In particular, we have investigated the stability of the RN-dS black hole and the SCC under such a perturbation by applying AIM method to calculate out the QNMs. When the coupling parameter is negative, there exists a purely imaginary unstable mode, indicating the instability of the black hole under consideration. When the coupling parameter is non-negative, such an instability disappears. On the other hand, with the increase of the non-negative coupling parameter, the violation of the SCC occurs at a larger critical charge ratio. Such an increase of the critical charge is nevertheless suppressed by the increase of the cosmological constant. In addition, we also find that different from the minimal coupling perturbation, the region for $\beta \geq 1$ emerges in the near extremal black hole for the non-minimal coupling, where the increase of the cosmological constant can make such a region enlarged toward a smaller value of the coupling parameter. The existence of this region implies that the resulting curvature can continuously cross the $\mathrm{CH}$.

It is noticed that here our discussion on the problem of SCC is only focused on the linear massless neutral scalar 
perturbation. However, a more general investigation on the problem of SCC in RN-dS spacetime still deserves further studies. One possible extension of our work is to examine the validity of SCC by studying the nonlinear effects of this nonminimal coupled scalar perturbation. On the other hand, more recently In the model that the scalar field is coupled with the Gauss-Bonnet term $[35,36]$, it was found that more solutions with different types of hairy BHs via the spontaneous scalarization can be constructed [37-40]. So as another model with scalar field non-minimally coupled to dS spacetime, studying the strong cosmic censorship for those scalarized hairy black holes could be another future direction.

Acknowledgements We are grateful to Hongbao Zhang for his helpful comments and suggestions. We also appreciate Zhiying Zhu for the helpful correspondence. This work is supported by the Natural Science Foundation of China under Grants no. 11705161 and Natural Science Foundation of Jiangsu Province under Grant no. BK20170481.

Data Availability Statement This manuscript has no associated data or the data will not be deposited. [Authors' comment: This is a theoretical work that no data need depositing.]

Open Access This article is distributed under the terms of the Creative Commons Attribution 4.0 International License (http://creativecomm ons.org/licenses/by/4.0/), which permits unrestricted use, distribution, and reproduction in any medium, provided you give appropriate credit to the original author(s) and the source, provide a link to the Creative Commons license, and indicate if changes were made.

Funded by SCOAP ${ }^{3}$.

\section{A A brief introduction of AIM}

We calculate the QNM frequency $\omega$ numerically by using the asymptotic iteration method (AIM) [41]. As such, we introduce a new variable $\xi=\frac{1}{r}$. Rewrite $\phi(r)$ in terms of the new variable $\xi$ as follows

$\phi(\xi)=\left(\xi_{+}-\xi\right)^{-\frac{i \omega}{2 \kappa_{+}}}\left(\xi-\xi_{c}\right)^{-\frac{i \omega}{2 \kappa_{c}}} \chi(\xi)$,

with $\xi_{+}=r_{+}^{-1}$ and $\xi_{c}=r_{c}^{-1}$, and recast Eq. (2.14) into the form as

$\chi^{\prime \prime}(\xi)=\lambda_{0}(\xi) \chi^{\prime}(\xi)+s_{0}(\xi) \chi(\xi)$.
Differentiating above equation, it is easy to obtain the iteration formula

$\chi^{(n+1)}(\xi)=\lambda_{n-1}(\xi) \chi^{\prime}(\xi)+s_{n-1}(\xi) \chi(\xi)$,

while these iterative parameters are given by

$\lambda_{n}(\xi)=\lambda_{n-1}^{\prime}(\xi)+s_{n-1}(\xi)+\lambda_{0}(\xi) \lambda_{n-1}(\xi)$,

$s_{n}(\xi)=s_{n-1}^{\prime}(\xi)+s_{0}(\xi) \lambda_{n-1}(\xi)$.

Then, the large $n$ asymptotic aspect and the quantization condition are introduced as

$\frac{s_{n}(\xi)}{\lambda_{n}(\xi)}=\frac{s_{n-1}(\xi)}{\lambda_{n-1}(\xi)} \equiv \beta(\xi)$,

$\delta_{n}=s_{n} \lambda_{n-1}-s_{n-1} \lambda_{n}=0$.

As shown above, the quantization condition imposes a termination of the iterations, which can read the QNMs of the system. In the development of the AIM method, an improved version [42] was proposed to bypass the iterative derivatives of each steps. The parameters $\lambda_{n}(\xi)$ and $s_{n}(\xi)$ are expanded around the point $\xi_{0}$

$\lambda_{n}(\xi)=\sum_{i=0}^{\infty} c_{n}^{i}\left(\xi-\xi_{0}\right)^{i}$,
$s_{n}(\xi)=\sum_{i=0}^{\infty} d_{n}^{i}\left(\xi-\xi_{0}\right)^{i}$.

while the expansion coefficients are given by

$c_{n}^{i}(\xi)=(i+1) c_{n-1}^{i+1}+d_{n-1}^{i}+\sum_{k=0}^{i} c_{0}^{k} c_{n-1}^{i-k}$,

$d_{n}^{i}(\xi)=(i+1) d_{n-1}^{i+1}+\sum_{k=0}^{i} d_{0}^{k} c_{n-1}^{i-k}$.

In these terms, the quantization condition is reread as

$d_{n}^{0} c_{n-1}^{0}-d_{n-1}^{0} c_{n}^{0}=0$.

Though the AIM has widely employed to compute quasinormal modes of black holes, it is still necessary to check its numerical accuracy in our model.To this end, we compare the results between the AIM and spectral method [43]. The

Table 6 The QNMs for AIM method and Spectral method when $M=1, \Lambda=0.14, Q / Q_{\max }=0.99, n=0, l=0$. We also calculate the relative deviation of the AIM method with the iteration $N=60$ relative to spectral method

\begin{tabular}{lllr}
\hline Coupling constant & AIM method & Spectral method & Relative deviation \\
\hline$\lambda=0$ & $0.309502-i 0.522089$ & $0.309502-i 0.522088$ & $\left(1.2 \times 10^{-6}, 5.5 \times 10^{-7}\right)$ \\
$\lambda=0.5$ & $1.085767-i 0.327964$ & $1.085767-i 0.327964$ & $\left(2.6 \times 10^{-9}, 5.7 \times 10^{-8}\right)$ \\
$\lambda=1$ & $1.561967-i 0.33067$ & $1.561967-i 0.33067$ & $\left(3.4 \times 10^{-9}, 1.2 \times 10^{-9}\right)$ \\
$\lambda=1.5$ & $1.925699-i 0.33257$ & $1.925699-i 0.33257$ & $\left(8.7 \times 10^{-9}, 7.4 \times 10^{-8}\right)$ \\
\hline
\end{tabular}


output for samples of model parameters is shown in Table 6 , the data of the two methods are almost consistent with six decimal places. Besides, we analyze the relative deviation of AIM with the iteration $N=60$ to spectral method, which ensures that our computation via AIM are precise and reliable enough.

\section{References}

1. S. Hod, Strong cosmic censorship in charged black-hole spacetimes: as strong as ever. Nucl. Phys. B 941, 636 (2019). arXiv: 1801.07261

2. V. Cardoso, J.L. Costa, K. Destounis, P. Hintz, A. Jansen, Quasinormal modes and strong cosmic censorship. Phys. Rev. Lett. 120, 031103 (2018). arXiv:1711.10502

3. D. Christodoulou, The formation of black holes in general relativity. In: The Twelfth Marcel Grossmann Meeting: On Recent Developments in Theoretical and Experimental General Relativity, Astrophysics and Relativistic Field Theories (In 3 Volumes), pp. 24-34, World Scientific (2008). arXiv:0805.3880

4. Z. Zhu, S.-J. Zhang, C.E. Pellicer, B. Wang, E. Abdalla, Stability of Reissner-Nordström black hole in de Sitter background under charged scalar perturbation. Phys. Rev. D 90, 044042 (2014). arXiv: 1405.4931

5. R.A. Konoplya, A. Zhidenko, Instability of higher dimensional charged black holes in the de-Sitter world. Phys. Rev. Lett. 103, 161101 (2009). arXiv:0809.2822

6. V. Cardoso, M. Lemos, M. Marques, On the instability of ReissnerNordstrom black holes in de Sitter backgrounds. Phys. Rev. D 80, 127502 (2009). arXiv:1001.0019

7. O.J.C. Dias, H.S. Reall, J.E. Santos, Strong cosmic censorship: taking the rough with the smooth. JHEP 10, 001 (2018). arXiv: 1808.02895

8. S. Hod, Quasinormal modes and strong cosmic censorship in nearextremal Kerr-Newman-de Sitter black-hole spacetimes. Phys. Lett. B 780, 221 (2018). arXiv:1803.05443

9. V. Cardoso, J.L. Costa, K. Destounis, P. Hintz, A. Jansen, Strong cosmic censorship in charged black-hole spacetimes: still subtle. Phys. Rev. D 98, 104007 (2018). arXiv:1808.03631

10. Y. Mo, Y. Tian, B. Wang, H. Zhang, Z. Zhong, Strong cosmic censorship for the massless charged scalar field in the ReissnerNordstrom-de Sitter spacetime. Phys. Rev. D 98, 124025 (2018). arXiv: 1808.03635

11. O.J.C. Dias, H.S. Reall, J.E. Santos, Strong cosmic censorship for charged de Sitter black holes with a charged scalar field. Class. Quantum Gravity 36, 045005 (2019). arXiv: 1808.04832

12. O.J.C. Dias, F.C. Eperon, H.S. Reall, J.E. Santos, Strong cosmic censorship in de Sitter space. Phys. Rev. D 97, 104060 (2018). arXiv:1801.09694

13. B. Ge, J. Jiang, B. Wang, H. Zhang, Z. Zhong, Strong cosmic censorship for the massless Dirac field in the Reissner-Nordstromde Sitter spacetime. JHEP 01, 123 (2019). arXiv:1810.12128

14. M. Rahman, S. Chakraborty, S. SenGupta, A.A. Sen, Fate of strong cosmic censorship conjecture in presence of higher spacetime dimensions. JHEP 03, 178 (2019). arXiv: 1811.08538

15. B. Gwak, Strong cosmic censorship under quasinormal modes of non-minimally coupled massive scalar field. Eur. Phys. J. C 79, 767 (2019). arXiv: 1812.04923

16. H. Liu, Z. Tang, K. Destounis, B. Wang, E. Papantonopoulos, H. Zhang, Strong cosmic censorship in higher-dimensional Reissner-Nordström-de Sitter spacetime. JHEP 03, 187 (2019). arXiv:1902.01865
17. B. Gwak, Quasinormal modes of massive scalar field with nonminimal coupling in higher-dimensional de Sitter Black Hole with single rotation. arXiv: 1903.11758

18. K. Destounis, Charged fermions and strong cosmic censorship. Phys. Lett. B 795, 211 (2019). arXiv:1811.10629

19. R. Luna, M. Zilhão, V. Cardoso, J.L. Costa, J. Natário, Strong cosmic censorship: the nonlinear story. Phys. Rev. D 99, 064014 (2019). arXiv:1810.00886

20. S.D. Deser, M.T. Grisaru, H. Pendleton (eds.), Lectures on elementary particles and quantum field theory, (Cambridge, MA, USA), MIT, Proceedings, 13th Brandeis University Summer Institute in Theoretical Physics (1970)

21. F.S. Accetta, D.J. Zoller, M.S. Turner, Induced gravity inflation. Phys. Rev. D 31, 3046 (1985)

22. J.-P. Uzan, Cosmological scaling solutions of nonminimally coupled scalar fields. Phys. Rev. D 59, 123510 (1999). arXiv:gr-qc/9903004

23. L. Amendola, Scaling solutions in general nonminimal coupling theories. Phys. Rev. D 60, 043501 (1999). arXiv:astro-ph/9904120

24. T. Chiba, Quintessence, the gravitational constant, and gravity. Phys. Rev. D 60, 083508 (1999). arXiv:gr-qc/9903094

25. F. Perrotta, C. Baccigalupi, S. Matarrese, Extended quintessence. Phys. Rev. D 61, 023507 (1999). arXiv:astro-ph/9906066

26. S. Randjbar-Daemi, A. Salam, J.A. Strathdee, On Kaluza-Klein cosmology. Phys. Lett. B 135, 388 (1984)

27. K.-I. Maeda, Stability and attractor in Kaluza-Klein cosmology 1. Class. Quantum Gravity 3, 233 (1986)

28. T. Appelquist, A. Chodos, Quantum effects in Kaluza-Klein theories. Phys. Rev. Lett. 50, 141 (1983)

29. V. Faraoni, Conformally coupled inflation. Galaxies 1, 96 (2013). arXiv: 1309.4900

30. B. Wang, E. Abdalla, R.B. Mann, Scalar wave propagation in topological black hole backgrounds. Phys. Rev. D 65, 084006 (2002). arXiv:hep-th/0107243

31. M. Chabab, H. El Moumni, S. Iraoui, K. Masmar, Behavior of quasinormal modes and high dimension RN-AdS black hole phase transition. Eur. Phys. J. C 76, 676 (2016). arXiv:1606.08524

32. P. Hintz, A. Vasy, Analysis of linear waves near the Cauchy horizon of cosmological black holes. J. Math. Phys. 58, 081509 (2017). arXiv: 1512.08004

33. K.A. Bronnikov, R.A. Konoplya, A. Zhidenko, Instabilities of wormholes and regular black holes supported by a phantom scalar field. Phys. Rev. D 86, 024028 (2012). arXiv:1205.2224

34. E.S.C. Ching, P.T. Leung, W.M. Suen, K. Young, Quasinormal mode expansion for linearized waves in gravitational system. Phys. Rev. Lett. 74, 4588 (1995). arXiv:gr-qc/9408043

35. H.O. Silva, J. Sakstein, L. Gualtieri, T.P. Sotiriou, E. Berti, Spontaneous scalarization of black holes and compact stars from a Gauss-Bonnet coupling. Phys. Rev. Lett. 120, 131104 (2018). arXiv: 1711.02080

36. D.D. Doneva, S.S. Yazadjiev, New Gauss-Bonnet black holes with curvature-induced scalarization in extended scalar-tensor theories. Phys. Rev. Lett. 120, 131103 (2018). arXiv:1711.01187

37. T. Ikeda, T. Nakamura, M. Minamitsuji, Spontaneous scalarization of charged black holes in the Scalar-Vector-Tensor theory, arXiv: 1908.09394

38. C.A.R. Herdeiro, E. Radu, N. Sanchis-Gual, J.A. Font, Spontaneous scalarization of charged black holes. Phys. Rev. Lett. 121, 101102 (2018). arXiv: 1806.05190

39. P.V.P. Cunha, C.A.R. Herdeiro, E. Radu, Spontaneously scalarized Kerr Black Holes in extended scalar-tensor-Gauss-Bonnet gravity. Phys. Rev. Lett. 123, 011101 (2019). arXiv:1904.09997

40. M. Minamitsuji, T. Ikeda, Spontaneous scalarization of black holes in the Horndeski theory. Phys. Rev. D 99, 104069 (2019). arXiv:1904.06572 
41. H.T. Cho, A.S. Cornell, J. Doukas, T.R. Huang, W. Naylor, A new approach to black hole quasinormal modes: a review of the asymptotic iteration method. Adv. Math. Phys. 2012, 281705 (2012). arXiv:1111.5024

42. H.T. Cho, A.S. Cornell, J. Doukas, W. Naylor, Black hole quasinormal modes using the asymptotic iteration method. Class. Quantum Gravity 27, 155004 (2010). arXiv:0912.2740
43. A. Jansen, Overdamped modes in Schwarzschild-de Sitter and a mathematica package for the numerical computation of quasinormal modes. Eur. Phys. J. Plus 132, 546 (2017). arXiv:1709.09178 